\title{
Variabilidad temporal de algunos parámetros físicos e hidroquímicos de un prado húmedo alto-andino del norte-centro de Chile
}

\author{
Temporal variability of some physical and hydrochemical parameters \\ of a high Andes wet-pasture of north-central Chile \\ Jorge Cepeda-Pizarro ${ }^{*}$
}

\begin{abstract}
RESUMEN
Dados los requerimientos, el agua dulce disponible en el norte de Chile está siendo cada vez más escasa. En las montañas andinas del norte centro de Chile, los prados húmedos o vegas son importantes reservorios de este recurso, constituyendo a la vez fuentes de forraje de buena calidad para el ganado trashumante y focos de vida silvestre. Por estos servicios ecosistémicos, los conflictos entre partes interesadas son frecuentes. En este trabajo se documenta la calidad del agua almacenada en una de estas vegas y la variabilidad temporal de algunos parámetros físicos y químicos de ella. Mediante pozos piezométricos y durante cuatro años, se analizó el comportamiento mensual y estacional de la cota piezométrica, $\mathrm{pH}$ y el contenido de SDT, Fe, Mn, Cu, As total, Fl total y sulfato total. El promedio mensual de la cota piezométrica varió entre $-3,8 \mathrm{~m}$ y $-2,5 \mathrm{~m}$; sin evidencia clara de un patrón estacional. Del mismo modo, dada la variabilidad de los parámetros químicos estudiados, no se observó un patrón estacional claro. Comparadas con la mayoría de las aguas subterráneas naturales, el agua contenida en la vega mostró valores altos de sulfato total, Fl total, As total, $\mathrm{Fe}, \mathrm{Mn}$ y Cu. No obstante, este nivel es consistente con el nivel observado en cuencas andinas más nortinas. Desde el punto de vista hidroquímico, el subsistema estudiado es altamente fluctuante. Las características físicas y químicas del agua son consistentes con las características geológicas de las montañas a través de las cuales las aguas fluyen. Datos preliminares sostienen la idea de que estos sistemas semiacuáticos pueden funcionar como filtros, mejorando la calidad del agua que sale del humedal.
\end{abstract}

Palabras clave: hidroquímica de desiertos, agua subterránea, ecosistemas de montaña, Andes desérticos, vegas andinas.

\section{ABSTRACT}

As a result of demand, fresh water in northern Chile is becoming increasingly scarce. In the Andes range of north-central Chile, wet-pastures or vegas are important reservoirs of this resource, along with being a source of good quality forage for transhumant livestock and wildlife spots. From these ecosystemic services, conflicts between interested parties are frequent. In this work the water quality and the temporal variability of some physical and chemical parameters of the water stored in one of these wet-pastures is documented. By using piezometric wells, the monthly and seasonal behavior of the piezometric level, $\mathrm{pH}, \mathrm{TDS}, \mathrm{Fe}, \mathrm{Mn}, \mathrm{Cu}$, total As, total Fl, and the content of total sulfate was monitored. The piezometric monthly mean varied between $-3.8 \mathrm{~m}$ and $-2.5 \mathrm{~m}$, without evidencing a clear seasonal pattern. Similarly, given the variability of studied chemical parameters, a clear seasonal pattern was not detected. Compared with most natural underground water, the content of total sulfate, total As, Fe, Mn and Cu was high. However, this level is consistent with that found in northernmost_Andean basins. From the hydrochemical viewpoint, the studied subsystem is highly fluctuating. The physical and chemical characteristics of the water are consistent with the geological characteristics of the mountains through which water flows.Preliminary information supports the idea that these systems may act as water-filters, improving the water-quality of the water that outflows from them.

Key words: desert hydrochemistry, underground water, alpine ecosystems, desert Andes, Andean peatlands.

\section{Introducción}

En los ecosistemas de montaña, el sustrato geológico por donde fluyen las aguas y la marcada estacionalidad del ambiente conforman un escenario donde las características físicas, químicas y climáticas imponen severas restricciones a la biota colonizadora (Hinden et al., 2005). Todo ello ocurre, además, en un marco temporal dominado por significativas variaciones interanuales

\footnotetext{
1 Departamento de Biología. Universidad de La Serena. Benavente 980. La Serena (Chile).

* Autor por correspondencia: jcepeda@userena.cl
} 
(Cooper et al., 2010). Para muchos organismos, esta dinámica ambiental significa verse enfrentados permanentemente a situaciones que, en términos de adaptabilidad, los fuerza a un estado de constante recolonización (Márquez et al., 2009).

En los Andes desérticos, los prados húmedos o vegas de altura son importantes reservorios de agua, recursos forrajeros para el ganado trashumante, vida silvestre y diversidad biológica (Osorio et al., 2006). En atención a estos servicios ecosistémicos existe interés ciudadano y gubernamental en la protección y conservación de estas unidades del paisaje andino (Contreras, 2002; Acosta \& Custodio, 2008; UNESCO, 2009). En los Andes del norte-centro de Chile, las vegas de altura pueden ser, según los niveles de humedad, pastizales continuos de vegetación azonal, prados semiinundados o sitios inundados con afloramiento y acumulación de agua y sales (Squeo et al., 2006a). En este trabajo se investigaron las características de algunos parámetros físicos y químicos del agua contenida en una vega semiinundada de la alta montaña de la hoya hidrográfica del río Elqui, una cuenca característica del desierto transicional de Chile. Los objetivos de este trabajo fueron 1) documentar la dinámica mensual y estacional del nivel freático; 2) caracterizar el agua contenida en la vega en términos de $\mathrm{pH}$, SDT, $\mathrm{Fe}, \mathrm{Mn}, \mathrm{Cu}$, As total, Fl total y sulfato total, y 3) describir la dinámica mensual y estacional de ellos. La importancia de abordar estos objetivos radica en que esta vega forma parte de la red proveedora de pastos frescos al ganado trashumante local (Osorio et al., 2006), a la vez que es parte de la red hidrológica del río Elqui, principal fuente de agua para el consumo humano, agrícola e industrial del área (DGA, 2004) y está sujeta a las influencias de la actividad minera que se realiza en su entorno (Oyarzún et al., 2006).

\section{Materiales y Métodos}

\section{Sitio de estudio}

El estudio se realizó en el prado húmedo llamado "vega Tambo-Puquíos" (VTP en adelante), ubicado en la sección andina de la hoya hidrográfica del río Elqui (Región de Coquimbo, Chile) (2945' S, $69^{\circ} 59^{\prime}$ O, Figura 1). VTP corresponde a una unidad paisajística de vegetación azonal. Está ubicada entre los 3.800 y $4.200 \mathrm{~m} \mathrm{snm}$, en un valle estrecho y profundo. Tiene una extensión de $\sim 6 \mathrm{~km}$ y una superficie de $\sim 10 \mathrm{~km}^{2}$ (Figura 2). Las condiciones climáticas e hidrológicas del área están descritas en Cepeda \& Novoa (2006) y Zavala (2006). VTP constituye una unidad paisajística formada por una cubierta continua de especies de plantas cespitosas rodeada por una estepa árida presente en las colinas cercanas. Incluye dentro de ella al estero Tambo-río Vacas Heladas, pozas interiores y depósitos de agua encerrados en la capa cespitosa de vegetación $(<1$ $\mathrm{m}$ de grosor) y en el sustrato mineral subyacente. En este estudio se investigaron algunas de las características físicas y químicas del agua contenida en la capa cespitosa y en el sustrato mineral subyacente, hasta los $10 \mathrm{~m}$ superficiales, sin hacer distinción entre ellas.

\section{Monitoreos y Análisis Hidroquímicos}

La información provino de una base de datos alimentada por una red de piezómetros instalados hasta la profundidad de $10 \mathrm{~m}$, en la sección Tambo de VTP (Figura 2, $3.980 \mathrm{~m} \mathrm{snm}$ ). Esta base fue solo recientemente liberada de la condición de uso restringido y privado por la compañía minera propietaria de ella. Para este trabajo se recurrió a los registros mensuales de la cota piezométrica, $\mathrm{pH}$, SDT, $\mathrm{Fe}, \mathrm{Mn}, \mathrm{Cu}$, As total, Fl total y sulfato total. La toma de muestras y los análisis fueron realizados por una empresa contratista especializada (SITAC, 1983-2001), según las normas y técnicas estándares establecidas para ello, en concordancia con APHA et al. (1985), bajo la supervisión de las instituciones estatales chilenas con competencia ambiental. El estudio abarcó el período 1996-1999. Por razones de comparación se monitoreó el estero Tambo-río Vacas Heladas y algunas de las pequeñas pozas presentes en la vega, ambos en la sección Tambo de VTP. Igualmente, la toma de muestras, los análisis químicos y la supervisión fueron realizados como se señala arriba.

\section{Resultados y Discusión}

$\mathrm{El} \mathrm{pH}$ del agua, en el período estudiado, varió entre ligeramente ácido $(\sim 5,0 \mathrm{upH})$ y neutro $(\sim 7,0$ upH). Se detectó la presencia de sulfato, $\mathrm{Fl}, \mathrm{As} \mathrm{Fe}$, $\mathrm{Cu}$ y Mn. Los niveles claramente más altos correspondieron a sulfato y SDT (Tabla 1). En general, el nivel más bajo del agua contenida en la vega se registró en mayo y junio $(-3,8 \mathrm{~m})$ y el más superficial en febrero $(-2,5 \mathrm{~m})$; la variabilidad asociada 


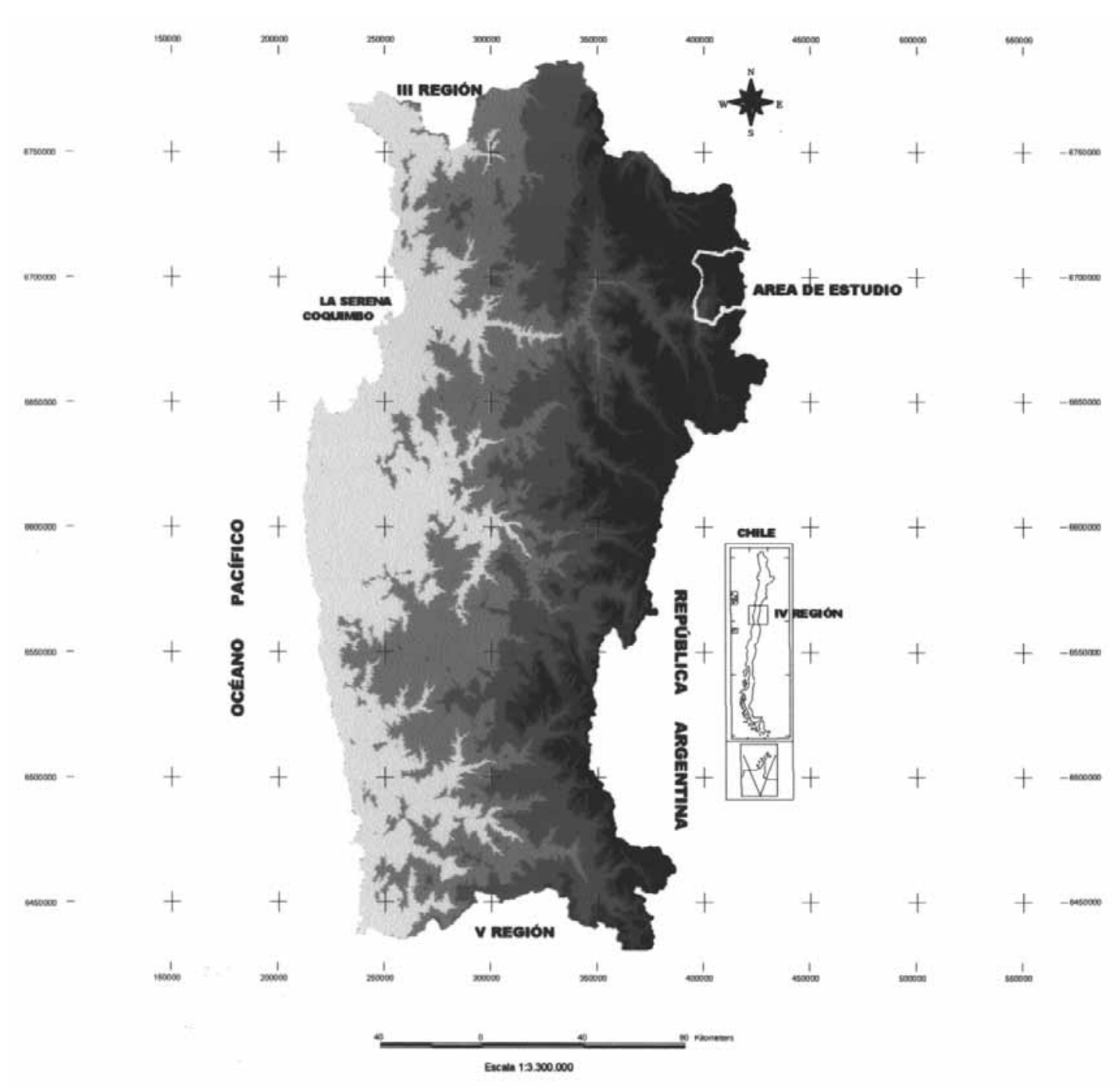

Figura 1. Ubicación del área de estudio ( $29^{\circ} 45^{\prime}$ S; $\left.69^{\circ} 59^{\prime} \mathrm{O}\right)$. La zona más oscura corresponde a las altitudes mayores encontradas en la hoya hidrográfica del río Elqui.

al promedio mensual fue baja (rango para el CV: 0,08-0,40). El promedio mensual del $\mathrm{pH}$ tomó un rango de 5,1 a 7,2; con variabilidad en torno a él baja (rango para el CV: 0,04-0,3). El promedio de SDT tomó un rango de 471,1 a $642,5 \mathrm{mg} / \mathrm{l}$; la variabilidad en torno al promedio fue baja (rango del CV: 0,080,45 ). La variabilidad en torno al promedio mensual de los componentes restantes, aunque presentes en niveles bajos a muy bajos, fue claramente más alta. Detalles de la dinámica mensual de los parámetros monitoreados se entrega en la Tabla 2.

Mientras el nivel estacional más bajo de la cota piezométrica fue $-4,5 \mathrm{~m}$, registrado en la primavera de 1999; el más alto correspondió a -2,2 m, registrado en la primavera de 1997. Llamativamente, el agua, independiente de la estación, mostró condiciones neutras al inicio del período examinado y condiciones ligeramente ácidas al final de él. El nivel más alto de SDT se registró en otoño, aunque un promedio muy bajo $(36,3 \mathrm{mg} / \mathrm{l})$ respecto del promedio general (780,9 mg/l) se registró en la primavera de 1996. El promedio estacional más alto del contenido de Fe se observó en primavera, aunque un promedio estacional muy alto $(11,4 \mathrm{mg} / \mathrm{l})$ y uno muy bajo $(0,4 \mathrm{mg} / \mathrm{l})$ comparados al promedio general $(6,0 \mathrm{mg} / \mathrm{l})$ fueron registrados en 1998 (invierno y primavera). 


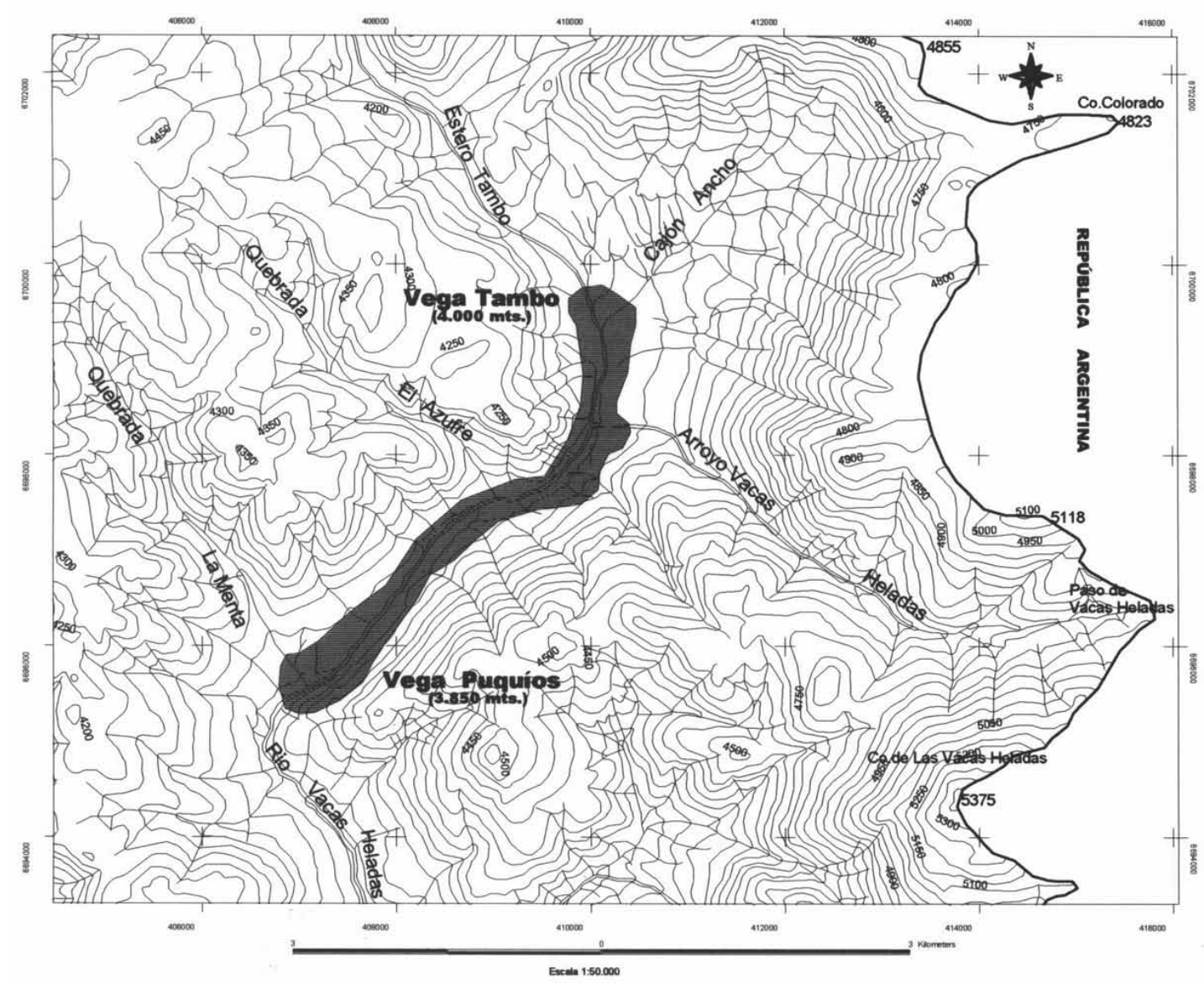

Figura 2: Rasgos fisiográficos de la vega Tambo Puquíos.

Tabla 1. Rango de valores de parámetros

limnéticos del agua en turba en VTP*.

\begin{tabular}{lc}
\hline Parámetro & Rango \\
\hline $\mathrm{pH}$ & $5-7$ \\
$\mathrm{SDT}$ & $471-654$ \\
$\mathrm{Mn}$ & $0,1-0,3$ \\
$\mathrm{Cu}$ & $0,03-0,20$ \\
$\mathrm{Fe}$ & $0,9-13,0$ \\
$\mathrm{As}$ total & $0,1-0,5$ \\
Fl total & $0,2-0,6$ \\
Sulfato total & $242-385$ \\
\hline
\end{tabular}

* VTP: vega Tambo-Puquíos, en sección Tambo (4.000 m snm, Figura 2). Valores en mg/l, excepto pH (upH).

El contenido estacional de Mn no mostró valores marginales, el promedio estacional más alto se registró en verano. Se registraron diferencias de dos órdenes de magnitud en el contenido estacional de $\mathrm{Cu}$; el contenido más alto se midió en verano $(0,1 \mathrm{mg} / \mathrm{l})$, respecto del resto de las estaciones $(0,05-0,07 \mathrm{mg} / \mathrm{l})$. El promedio estacional más alto del contenido de As se registró en verano $(0,37 \mathrm{mg} / \mathrm{l})$; no se observaron 
Tabla 2. Promedios mensuales $( \pm \mathrm{DS})$ de parámetros descriptivos del agua en turba en VTP*

Serie de tiempo: 1996-99. Valores en mg/l, excepto cota piezométrica (m) y pH (upH).

\begin{tabular}{|c|c|c|c|c|c|c|c|c|c|c|c|c|}
\hline \multirow{2}{*}{ Parámetro } & \multicolumn{12}{|c|}{ Mes } \\
\hline & E & $\mathrm{F}$ & M & A & M & $\mathrm{J}$ & $\mathrm{J}$ & A & $\mathrm{S}$ & $\mathrm{O}$ & $\mathrm{N}$ & $\mathrm{D}$ \\
\hline Cota piezométrica & $\begin{array}{r}-3,4 \\
(0,4)\end{array}$ & $\begin{array}{l}-2,5 \\
(1,0)\end{array}$ & $\begin{array}{l}-3,7 \\
(0,6)\end{array}$ & $\begin{array}{c}-3,4 \\
(0,4)\end{array}$ & $\begin{array}{c}-3,8 \\
(0,3)\end{array}$ & $\begin{array}{c}-3,3 \\
(0,4)\end{array}$ & $\begin{array}{l}-3,8 \\
(0,2)\end{array}$ & $\begin{array}{l}-3,3 \\
(0,3)\end{array}$ & $\begin{array}{l}-3,3 \\
(0,3)\end{array}$ & $\begin{array}{l}-3,5 \\
(1,3)\end{array}$ & $\begin{array}{c}-3,1 \\
(0,8)\end{array}$ & $\begin{array}{l}-3,3 \\
(0,6)\end{array}$ \\
\hline $\mathrm{pH}$ & $\begin{array}{c}6,9 \\
(0,3)\end{array}$ & $\begin{array}{c}5,1 \\
(1,6)\end{array}$ & $\begin{array}{c}7,1 \\
(0,8)\end{array}$ & $\begin{array}{c}6,7 \\
(0,6)\end{array}$ & $\begin{array}{c}6,9 \\
(0,3)\end{array}$ & $\begin{array}{c}7,0 \\
(0,4)\end{array}$ & $\begin{array}{c}6,9 \\
(0,3)\end{array}$ & $\begin{array}{c}7,1 \\
(0,5)\end{array}$ & $\begin{array}{c}6,7 \\
(0,3)\end{array}$ & $\begin{array}{c}6,8 \\
(0,3)\end{array}$ & $\begin{array}{c}6,8 \\
(0,6)\end{array}$ & $\begin{array}{c}7,2 \\
(0,7)\end{array}$ \\
\hline SDT & $\begin{array}{l}567,1 \\
(44,7)\end{array}$ & $\begin{array}{c}471,1 \\
(191,5)\end{array}$ & $\begin{array}{c}567,2 \\
(169,0)\end{array}$ & $\begin{array}{c}654,0 \\
(165,8)\end{array}$ & $\begin{array}{c}642,5 \\
(148,8)\end{array}$ & $\begin{array}{l}517,5 \\
(94,1)\end{array}$ & $\begin{array}{l}541,9 \\
(55,1)\end{array}$ & $\begin{array}{l}569,5 \\
(73,9)\end{array}$ & $\begin{array}{l}564,9 \\
(90,1)\end{array}$ & $\begin{array}{c}623,9 \\
(281,1)\end{array}$ & $\begin{array}{c}628,0 \\
(150,7)\end{array}$ & $\begin{array}{l}514,2 \\
(59,7)\end{array}$ \\
\hline $\mathrm{Mn}$ & $\begin{array}{c}0,3 \\
(0,3)\end{array}$ & $\begin{array}{c}0,3 \\
(0,2)\end{array}$ & $\begin{array}{c}0,2 \\
(0,2)\end{array}$ & $\begin{array}{c}0,2 \\
(0,0)\end{array}$ & $\begin{array}{c}0,1 \\
(0,1)\end{array}$ & $\begin{array}{c}0,1 \\
(0,1)\end{array}$ & $\begin{array}{c}0,1 \\
(0,1)\end{array}$ & $\begin{array}{c}0,1 \\
(0,0)\end{array}$ & $\begin{array}{c}0,3 \\
(0,3)\end{array}$ & $\begin{array}{c}0,2 \\
(0,2)\end{array}$ & $\begin{array}{c}0,3 \\
(0,3)\end{array}$ & $\begin{array}{c}0,2 \\
(0,2)\end{array}$ \\
\hline $\mathrm{Cu}$ & $\begin{array}{c}0,18 \\
(0,1)\end{array}$ & $\begin{array}{c}0,11 \\
(0,1)\end{array}$ & $\begin{array}{r}0,05 \\
(0,0)\end{array}$ & $\begin{array}{r}0,03 \\
(0,0)\end{array}$ & $\begin{array}{r}0,04 \\
(0,0)\end{array}$ & $\begin{array}{c}0,12 \\
(0,1)\end{array}$ & $\begin{array}{r}0,04 \\
(0,0)\end{array}$ & $\begin{array}{r}0,03 \\
(0,0)\end{array}$ & $\begin{array}{r}0,04 \\
(0,0)\end{array}$ & $\begin{array}{r}0,05 \\
(0,0)\end{array}$ & $\begin{array}{c}0,06 \\
(0,0)\end{array}$ & $\begin{array}{r}0,18 \\
(0,2)\end{array}$ \\
\hline $\mathrm{Fe}$ & $\begin{array}{c}6,5 \\
(6,8)\end{array}$ & $\begin{array}{c}6,4 \\
(4,8)\end{array}$ & $\begin{array}{c}3,1 \\
(1,9)\end{array}$ & $\begin{array}{c}2,4 \\
(0,6)\end{array}$ & $\begin{array}{c}2,6 \\
(2,2)\end{array}$ & $\begin{array}{c}4,0 \\
(0,9)\end{array}$ & $\begin{array}{c}6,3 \\
(5,7)\end{array}$ & $\begin{array}{c}0,9 \\
(0,6)\end{array}$ & $\begin{array}{c}1,9 \\
(2,0)\end{array}$ & $\begin{array}{c}5,6 \\
(3,5)\end{array}$ & $\begin{array}{l}13,0 \\
(8,4)\end{array}$ & $\begin{array}{c}5,8 \\
(4,0)\end{array}$ \\
\hline As total & $\begin{array}{c}0,3 \\
(0,4)\end{array}$ & $\begin{array}{c}0,3 \\
(0,2)\end{array}$ & $\begin{array}{c}0,1 \\
(0,0)\end{array}$ & $\begin{array}{c}0,3 \\
(0,3)\end{array}$ & $\begin{array}{c}0,1 \\
(0,0)\end{array}$ & $\begin{array}{c}0,2 \\
(0,0)\end{array}$ & $\begin{array}{c}0,3 \\
(0,2)\end{array}$ & $\begin{array}{c}0,3 \\
(0,3)\end{array}$ & $\begin{array}{c}0,4 \\
(0,2)\end{array}$ & $\begin{array}{c}0,2 \\
(0,2)\end{array}$ & $\begin{array}{c}0,5 \\
(0,3)\end{array}$ & $\begin{array}{c}0,3 \\
(0,2)\end{array}$ \\
\hline Fl total & $\begin{array}{c}0,4 \\
(0,1)\end{array}$ & $\begin{array}{c}0,2 \\
(0,1)\end{array}$ & $\begin{array}{c}0,3 \\
(0,1)\end{array}$ & $\begin{array}{c}0,5 \\
(0,3)\end{array}$ & $\begin{array}{c}0,4 \\
(0,1)\end{array}$ & $\begin{array}{c}0,4 \\
(0,0)\end{array}$ & $\begin{array}{c}0,4 \\
(0,2)\end{array}$ & $\begin{array}{c}0,6 \\
(0,1)\end{array}$ & $\begin{array}{r}0,4 \\
(0,1\end{array}$ & $\begin{array}{c}0,4 \\
(0,3)\end{array}$ & $\begin{array}{c}0,5 \\
(0,2)\end{array}$ & $\begin{array}{c}0,3 \\
(0,1)\end{array}$ \\
\hline Sulfato total & $\begin{array}{r}291,0 \\
(5,9)\end{array}$ & $\begin{array}{c}265,8 \\
(151,7)\end{array}$ & $\begin{array}{c}358,9 \\
(90,1)\end{array}$ & $\begin{array}{l}369,6 \\
(86,5)\end{array}$ & $\begin{array}{c}384,5 \\
(125,0)\end{array}$ & $\begin{array}{l}338,5 \\
(65,0)\end{array}$ & $\begin{array}{l}316,5 \\
(23,0)\end{array}$ & $\begin{array}{l}242,0 \\
(39,6)\end{array}$ & $\begin{array}{l}314,3 \\
(18,0)\end{array}$ & $\begin{array}{l}323,7 \\
(64,7)\end{array}$ & $\begin{array}{l}344,1 \\
(79,9)\end{array}$ & $\begin{array}{l}309,9 \\
(76,1)\end{array}$ \\
\hline
\end{tabular}

* VTP: como en la Tabla 1.

promedios estacionales marginales. El promedio estacional más alto del contenido de Fl se registró en verano $(0,47 \mathrm{mg} / \mathrm{l})$; no se observaron promedios estacionales marginales. El promedio estacional más alto del contenido de sulfato se registró en otoño $(353,4 \mathrm{mg} / \mathrm{l})$; no se observaron promedios estacionales marginales. El detalle de esta dinámica se entrega en la Tabla 3.

A pesar de la importancia que puede tener el agua contenida en el subsistema freático en la estructura y dinámica de los ecosistemas lóticos y lénticos de ambientes áridos (Álvarez-Cobelas, 2006), particularmente en el caso de los humedales andinos (Squeo et al., 2006a; Ginocchio et al., 2008), la información pública es muy reducida; esta situación no quiere decir que el conocimiento e información no exista. Por el contrario, debido al auge de la minería experimentado en el norte-centro y norte de Chile, la escasez absoluta del agua en estos territorios, las exigencias ambientales y los movimientos ciudadanos cada vez más empoderados, esta información existe, sólo que es de uso reservado y compleja en su interpretación. Este trabajo constituye el primer esfuerzo de descripción, documentación e interpretación de la hidroquímica de un ambiente húmedo enclavado en la cordillera de los Andes del desierto transicional de Chile.
La vega estudiada forma parte, en su sección altoandina, de la hoya hidrográfica del río Elqui (DGA, 2004; Strauch et al., 2006). Esta es una cuenca nival; provee agua tanto para una pujante fruticultura de exportación y minería, como para una población humana en constante crecimiento, principalmente concentrada en la conurbanación costera de La Serena-Coquimbo (ODEPA, 2011). Constituye, a su vez, un reservorio de recursos forrajeros y unidad de vida silvestre (Cepeda et al., 2006; Squeo et al., 2006b). La vega Tambo-Puquíos corresponde a pastos cespitosos mantenidos por precipitación nival, drenaje superficial y subterráneo. En este sistema, el agua se distribuye y mantiene tanto en las capas orgánicas superficiales como en el suelo mineral subyacente (Zavala, 2006). Al respecto, el cambio temporal observado en el nivel piezométrico es más drástico a aquel reportado por Squeo et al. (2006a). Este hecho, junto a la ausencia de diferencias estacionales, sea probablemente la consecuencia de la extracción continua de agua por parte de la faena minera alguna vez realizada en el área (Oyarzún et al., 2006).

En general, la composición química del agua del estanco estudiado es consistente con la naturaleza geológica del sustrato por donde las aguas drenan antes de ser parte de la vega (Oyarzún et al., 2006). 


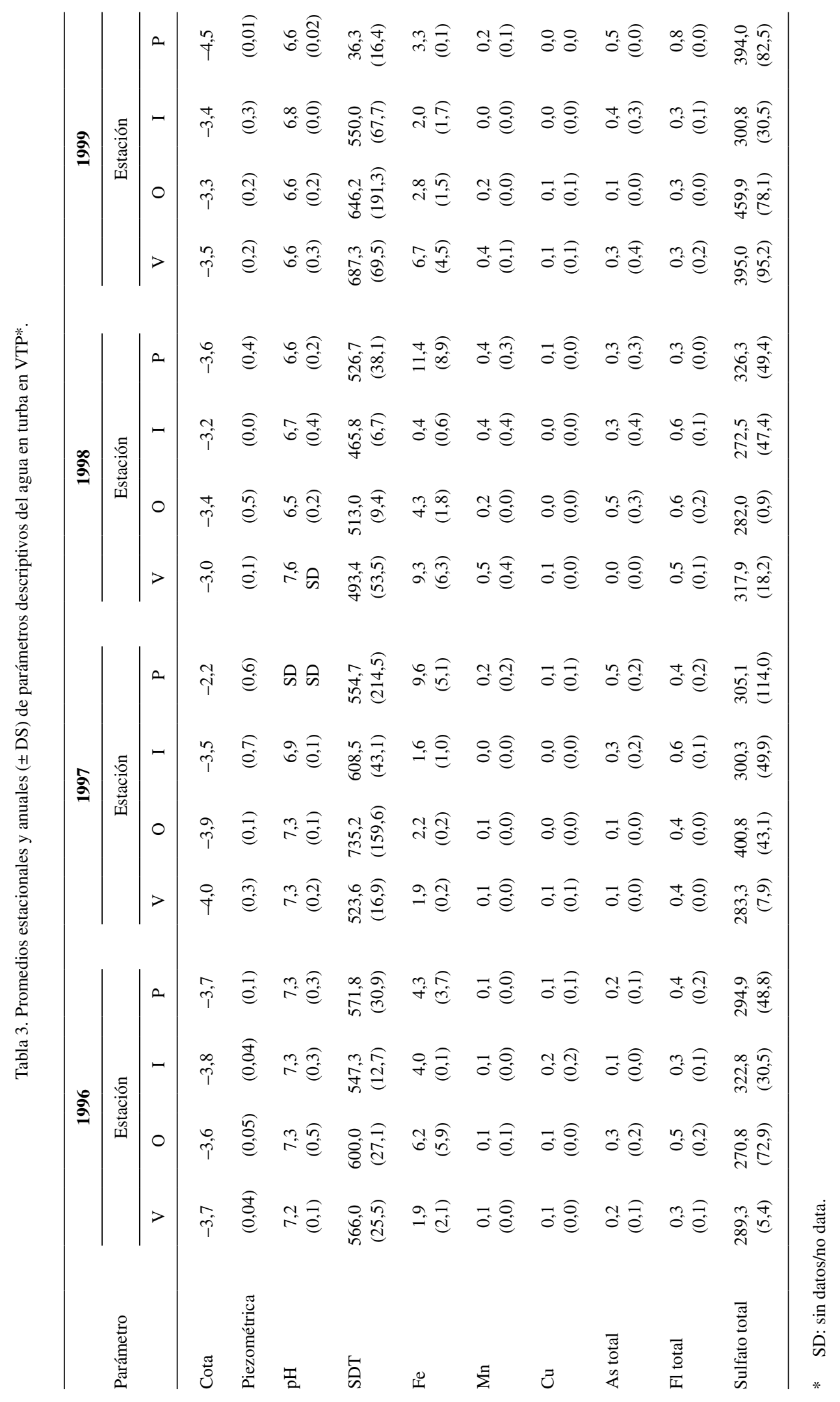


La variabilidad hidroquímica temporal observada en este trabajo es consistente con estudios previos realizados en ambientes acuáticos de alta montaña (p. e., Hinden et al., 2005; Ginocchio et al., 2008). Probablemente, esta variabilidad impidió detectar un patrón temporal.

El subsistema examinado en este trabajo muestra diversas diferencias con ambientes subterráneos de tierras bajas y de bofedales del Norte Grande de Chile. En general, las aguas subterráneas de bofedales son alcalinas, con un rango típico de salinidad entre 1.000 y $7.000 \mathrm{mg} / \mathrm{l} \mathrm{SDT}$, contenido de sulfato total entre 30-90 mg/l, y valores altos de nitrato (Anónimo, 1977). Por el contrario, el agua de la vega Tambo-Puquíos tiene $\mathrm{pH}$ ligeramente ácido, es relativamente baja en SDT, pero alta en sulfato total. Aunque no se tiene información respecto del contenido de nitrógeno en ninguna de sus formas en el agua freática de la vega Tambo-Puquíos, análisis preliminares del contenido de nitrato en el agua del estero Tambo-río Vacas Heladas, curso de agua incluido en la vega, muestran valores altos (p. e., 13,6 mg/l).

El sulfato es un componente principal del agua subterránea natural, el Fe y el Fl son componentes secundarios y el $\mathrm{Mn}$, As total y $\mathrm{Cu}$, componentes menores. $\mathrm{Al}$ respecto, el rango del promedio mensual del Fl total está dentro de lo encontrado en aguas naturales subterráneas (Chapman, 1996). El rango del sulfato total observado en este estudio se halla en el límite superior de lo observado en aguas naturales subterráneas y es consistente con lo encontrado en vegas de cuencas nortinas (Chapman, 1996; Anónimo, 1977). Por su parte, el contenido mensual promedio de As total supera el valor máximo reportado para aguas subterráneas naturales, pero se encuentra dentro del rango observado en aguas subterráneas de cuencas andinas nortinas (Anónimo, 1977; Queirolo et al., 2000). Los niveles de $\mathrm{Fe}, \mathrm{Mn}$ y $\mathrm{Cu}$ superan los niveles superiores para aguas subterráneas naturales (Chapman, 1996).

Algunos autores, entre ellos Minckley et al. (2013), han señalado que los humedales de regiones áridas podrían cumplir funciones tan o más importante que aquellos ubicados en climas mésicos o tropicales. Para las regiones andinas áridas o semiáridas, dado el sustrato geológico por donde fluyen las aguas, una de estas funciones sería la purificación del agua. Aunque los datos son preliminares, estos muestran diferencias entre los compartimientos monitoreados (Tabla 4). Por ejemplo, el nivel promedio general es más alto en las pozas interiores; el $\mathrm{pH}$ se observa cercano a la neutralidad en el agua almacenada en la matriz; este decrecimiento del nivel también se observó en el contenido de Mn, Fl y sulfatos. Los datos no son concluyentes respecto de $\mathrm{Cu}$ y $\mathrm{Fe}$. Este papel debiera ser estudiado más profundamente si se quieren fundamentar debidamente planes de conservación y manejo sustentable de estas unidades paisajísticas de los Andes del nortecentro de Chile.

Tabla 4. Niveles promedio ( \pm DS) de SDT, pH, metales disueltos e inorgánicos no metales en tres tipos de hábitats acuáticos presentes en la vega Tambo-Puquíos. (Cuenca del Río del Toro, Cordillera de Doña Ana, IV Región, Coquimbo). Valores en $\mathrm{mg} / \mathrm{l}$, excepto $\mathrm{pH}$.

\begin{tabular}{|c|c|c|c|}
\hline \multirow{2}{*}{ Parámetro } & \multicolumn{3}{|c|}{ Hábitat } \\
\hline & Puquíos & Estero Tambo & Agua almacenada \\
\hline \multicolumn{4}{|l|}{ 1. Físicos } \\
\hline SDT (mg/l) & 1229,50 & SD & $583,61 \pm(68,68)$ \\
\hline 2. Químicos & SD & SD & \\
\hline $\mathrm{pH}$ & $8,51 \pm(0,51)$ & $6,41 \pm(0,84)$ & $6,96 \pm(0,32)$ \\
\hline As total & 0,26 & $0,32 \pm(0,22)$ & $0,25 \pm(0,03)$ \\
\hline $\mathrm{Cu}$ & 0,02 & $0,16 \pm(0,09)$ & $0,07 \pm(0,02)$ \\
\hline $\mathrm{Fe}$ & 0,33 & $8,47 \pm(10,36)$ & $4,85 \pm(1,55)$ \\
\hline $\mathrm{Mn}$ & 2,43 & $1,92 \pm(2,36)$ & $0,20 \pm(0,13)$ \\
\hline Fl total & $1,31 \pm(0,30)$ & $0,87 \pm(0,42)$ & $0,41 \pm(0,05)$ \\
\hline Sulfato total & $975,64 \pm(36,31)$ & $501,86 \pm(442,06)$ & $325,48 \pm(41,40)$ \\
\hline
\end{tabular}




\section{Conclusiones}

No se detectaron patrones temporales asociados a la cota piezométrica y al comportamiento de SDT, sulfato total, Fl total, As total, Fe, Mn y $\mathrm{Cu}$. Comparada con aguas subterráneas naturales, el agua de la vega mostró valores altos de estos componentes; no obstante, este nivel es consistente con el nivel observado en cuencas andinas más nortinas. Se observó que las características químicas del agua se corresponden con las características geológicas de las montañas a través de las cuales las aguas fluyen. Con excepción del $\mathrm{pH}$, los niveles de SDT, sulfato total, Fl total, As total, $\mathrm{Fe}, \mathrm{Mn}$ y $\mathrm{Cu}$ fueron temporalmente muy fluctuantes, no pudiéndose observar un patrón temporal. Los valores más fluctuantes correspondieron a los promedios mensuales. Información preliminar muestra un posible papel purificador del compartimiento matriz cespitosasustrato mineral subyacente sobre los componentes químicos contenidos en el agua. Esta sospecha amerita un estudio más focalizado.

\section{Agradecimientos}

Se agradece el acceso a la base de datos hidroquímicos de la subcuenca del río Vacas Heladas, cuenca del río del Toro, en la alta montaña de la hoya hidrográfica del río Elqui (Chile), de propiedad de la ex-Compañía Minera El Indio. La preparación de esta publicación fue financiada por la Dirección de Investigación de la Universidad de La Serena, La Serena (Chile).

\section{Literatura Citada}

Acosta, O.; Custodio, E.

2008. Impactos ambientales de las extracciones de agua subterránea en el salar de Huayco (norte de Chile). Boletín Geológico Minero, 119: 33-50.

Álvarez-Cobelas, M.

2006. Groundwater mediated limnology in Spain. Limnetica, 25: 1-2, 107-122.

Anónimo.

1977. Investigación de recursos hidráulicos en el Norte Grande de Chile: Hidrogeología de la Segunda Región, con especial referencia a las zonas investigadas (Informe Técnico). Investigación de Recursos Hidráulicos en el Norte Grande (CHI 69/535. Volumen I). Santiago, Chile.

APHA; AWWA; WPCF.

1985. Standard Methods for the Examination of Water and Wastewater. American Public Health Association American (APHA), American Water Works Association (AWWA) \& Water Pollution Control Federation (WPCF). 16th edition. Washington D.C.

Cepeda P.J.; Novoa J.J.

2006. La cordillera alto andina del Valle del Elqui. En: Geoecología de los Andes desérticos. La alta montaña del valle del Elqui. Cepeda P., J. (ed.). Ediciones Universidad de La Serena. La Serena, Chile, pp. 39-66.

Cepeda P.J.; Squeo P.F.; Cortés M.A.; Oyarzún M.J.; Zavala H.H. 2006. La biota del humedal Tambo-Puquíos. En: Geoecología de los Andes desérticos. La alta montaña del valle del Elqui. Cepeda P., J. (ed.) Ediciones Universidad de La Serena. La Serena, Chile, pp. 241-284.

Chapman, D. (ed.).

1996. Water quality assessments. A guide to use of biota, sediments and water in environmental monitoring. UNESCO/ WHO/UNEP. E \& FN Spon, an imprint of Chapman and Hall. Cambridge University Press. $2^{\text {nd }}$ Edition. Great Britain.
Contreras, J.P.

2002. Norte de Chile: conservación de humedales alto-andinos para un desarrollo productivo sustentable. Revista Ambiente y Desarrollo, XVIII: 125-131.

Cooper, D.J.; Wolf, E.C.; Colson, C.; Vering, W.; Granda, A.; Meyer, $\mathrm{M}$.

2010. Alpine peatlands of the Andes, Cajamarca, Peru. Arctic, Antarctic, and Alpine Research, 42: 1, 19-33.

DGA.

2004. Diagnóstico y clasificación de los cuerpos de agua según objetivos de calidad. Cuenca del río Elqui (Informe Técnico). Dirección General de Aguas. Ministerio de Obras Públicas. Gobierno de Chile. Santiago, Chile.

Ginocchio, R.; Hepp, J.; Bustamante, E.; Silva, Y.; De La Fuente, L.M.; Casale, J.F.; De La Harpe, J.P.; Urrestarazu, P.; Anic, V.; G. Montenegro.

2008. Importance of water quality on plant abundance and diversity in high-alpine meadows of the Yerba Loca Natural Sanctuary at the Andes of north-central Chile. Revista Chilena de Historia Natural, 81: 469-488.

Hinden, H.; Oertli, B.; Menetrey, N.; Sager, L.; Lachavannen, J.B. 2005. Alpine pond diversity: what are the related environmental variables? Aquatic Conservation 15 (6): 613-624.

Márquez-García, M.; Vila, I.; Hinojosa, L.F.; Méndez, M.A.; Carvajal, L.; Sabando, M.C.

2009. Distribution and seasonal fluctuations in the aquatic biodiversity of the southern Altiplano. Limnologica, 39: 314-318.

Minckley, T.A.; Turner, D.S.; Weinstein, S.R.

2013. The relevance of wetland conservation in arid regions: a re-examination of vanishing communities in the American Southwest. Journal of Arid Environments, 88: 213-221.

ODEPA.

2011. Catastro frutícola. Principales resultados. IV Región de Coquimbo. Oficina de Estudios y Políticas Agrarias. 
Ministerio de Agricultura. Gobierno de Chile. Santiago, Chile.

Osorio B.R.; Cabezas C.R.; Reyes S.H.; Álvarez L.P.; Koné, T. 2006. Humedales altoandinos de la IV Región. En: Geoecología de los Andes desérticos. La altamontaña del valle del Elqui. Cepeda P., J. (ed.). Ediciones Universidad de La Serena. La Serena, Chile, pp. 153-239.

Oyarzún, R.; Guevara, S.; Oyarzún, J.; Lillo, J.; Maturana, H.; Higueras., $\mathrm{P}$.

2006. The As-contaminated Elqui river basin: a long lasting perspective (1975-1995) covering the initiation and development of Au-Cu-As mining in the high Andes of northern Chile. Environmental Geochemistry and Health, 28: 431-443.

Queirolo, F.; Stegen, S.; Mondaca, J.; Cortés, R.; Rojas, R.; Contreras, C.; Muñoz, L.; Schwuger, M.J.; Ostapczuk, P. 2000. Total arsenic, lead, cadmium, copper, and zinc in some salt rivers in the northern Andes of Antofagasta, Chile. Science of The Total Environment, 255: 1-3, 85-95.

SITAC.

1983-2001. Programa de control de calidad de agua. Cuenca del Río Elqui. IV Región de Coquimbo (Informes Técnicos Anuales 1982-2000). Compañía Minera El Indio. Santiago, Chile.
Squeo, F.; Warner, B.; Aravena, G.; Espinoza, D. 2006a. Bofedales: high altitude peatlands of the central Andes. Revista Chilena de Historia Natural, 79: 245-255.

Squeo, F.; Ibacache, E.; Warner, B.; Espinoza, D.; Aravena, R.; Gutiérrez, J. R.

2006b. Productividad y diversidad florística de la vega Tambo. Cordillera de doña Ana. En: Geoecología de los Andes desérticos. La alta montaña del valle del Elqui. Cepeda P., J. (ed.): Ediciones Universidad de La Serena. La Serena, Chile, pp. 323-354.

Strauch, G.; Oyarzún, J.; Fiebig-Wittmaack, M.; González, E.; Weise, S.M.

2006. Contributions of the different water sources to the Elqui river runoff (northern Chile) evaluated by H/O isotopes. Isotopes in Environmental and Health Studies, 42: 303-322. UNESCO.

2009. Minería sustentable en zonas áridas. Aportes temáticos del proyecto CAMINAR (Documento Técnico del PHI-LAC $\mathrm{N}^{\circ}$ 15). CAZALAC. La Serena, Chile.

Zavala, $\mathrm{H}$.

2006. Hidrología del humedal Tambo-Puquíos. En: Geoecología de los Andes desérticos. La alta montaña del valle del Elqui. Cepeda P., J. (ed.). Ediciones Universidad de La Serena. La Serena, Chile, pp. 285-322. 
